\title{
ASYMPTOTIC BEHAVIOR OF SOLUTIONS OF THE HAMER EQUATION
}

\author{
A. A. $\mathrm{SOLOV}^{\prime} \mathrm{EV}$
}

\begin{abstract}
In a preceding paper the leading term was found for the asymptotics as $t \rightarrow+\infty$ of the solution of the initial problem for the Hamer equation, which is a simplest model for the motion of a radiating gas. Here, the second asymptotic term is constructed. It is proved that this term is proportional to the second term of the asymptotics of the solution of the initial problem for the Burgers equation.
\end{abstract}

\section{$\S 1$. INTRODUCTION}

The one-dimensional movement of a radiating gas is described by a system of the Euler equations that takes the heat radiation into account; see [1. As a simplest model of that full system, the following system of Hamer's equations (see [2]) is known:

$$
\left\{\begin{array}{l}
u_{t}+u \partial_{x} u+\partial_{x} q=0, \\
-\partial_{x}^{2} q+q+\partial_{x} u=0,
\end{array}\right.
$$

where $u(x, t)$ and $q(x, t), x \in R, t \in R_{+}$, are the velocity and the heat flow of the gas. We consider system (1.1) with the initial condition

$$
u(x, 0)=u_{0}(x) .
$$

Problem (1.1), (1.2) can be reduced to the form

$$
\left\{\begin{array}{l}
u_{t}+u \partial_{x} u+u-K u=0, \\
u(x, 0)=u_{0}(x)
\end{array}\right.
$$

where

$$
(K u)(x, t)=(K * u)(x, t) \text { with } K(x)=\frac{1}{2} e^{-|x|} .
$$

Kawashima proved (see [3]) that, even for smooth initial data, the solution of system (1.3) can become discontinuous in finite time. Therefore, one cannot expect the existence of a global solution.

However, later, Kawashima and Tanaka proved in [1] that a global smooth solution exists indeed under the assumption that the initial function is sufficiently small.

Before stating this results, we introduce some notation.

As usual, the symbol $\mathcal{F}[f]$ denotes the Fourier transform of a function $f$, given by

$$
\mathcal{F}[f](\xi)=\widehat{f}(\xi)=\int_{-\infty}^{\infty} e^{-i x \xi} f(x) d x .
$$

The inverse Fourier transformation is denoted by $\mathcal{F}^{-1}$. Next, $L^{p}=L^{p}(R), 1 \leq p \leq \infty$, is the usual Lebesgue space of functions on $R$, with the norm $\|\cdot\|_{L^{p}}$.

2010 Mathematics Subject Classification. Primary 35G55.

Key words and phrases. Asymptotic behavior, initial problem, radiating gas.

Supported by RFBR (grant no. 12-01-00259-a). 
For a nonnegative integer $s$, the symbol $H^{s}=H^{s}(R)$ denotes the Sobolev space of $L^{2}$-functions equipped with the norm

$$
\|f\|_{H^{s}}=\left(\sum_{k=0}^{s}\left\|\partial_{x}^{k} f\right\|_{L^{2}}^{2}\right)^{1 / 2} .
$$

Here $\partial_{x}^{k}$ stands for the derivative of order $k$ with respect to the variable $x \in R$. By $C^{k}\left(H^{s}(R), I\right)$ we denote the space of $k$ times continuously differentiable functions defined on $I$ and taking values in $H^{s}(R)$.

Throughout, we denote various positive constants by $C$ (possibly, with indices).

Now we formulate the theorem proved in [1] and claiming the existence of a global solution.

Theorem 1 (S. Kawashima and Y. Tanaka). There exist two positive constants $\delta_{0}$ and $C$ such that if

$$
\left\|u_{0}\right\|_{H^{s}(R)} \leq \delta_{0}
$$

then problem (1.1), (1.2) has a unique global solution $(u(x, t), q(x, t))$, satisfying the conditions

$$
\begin{aligned}
& u \in C\left(H^{s}(R),[0, \infty)\right) \cap C^{1}\left(H^{s-1}(R),[0, \infty)\right), \\
& q \in C\left(H^{s+1}(R),[0, \infty)\right) .
\end{aligned}
$$

Also, this solution $(u, q)$ obeys the following energy estimate uniform in $t$ :

$$
\|u(t)\|_{H^{s}}^{2}+\int_{0}^{t}\left(\|u(\tau)\|_{H^{s-1}}^{2}+\|q(\tau)\|_{H^{s+1}}^{2}\right) d \tau \leq C \delta_{0}^{2}
$$

The behavior of the solution $(u(x, t), q(x, t))$ as $t \rightarrow \infty$ of system (1.1) with initial data belonging to $H^{s}(R) \cap L^{1}(R)$ was studied in [4].

Theorem 2 (S. Kawashima and Y. Liu). Suppose that $u_{0} \in H^{s}(R) \cap L^{1}(R)$ with $s \geq 3$. There exists a small positive constant $\delta_{1}$ such that if

$$
E_{1}=\left\|u_{0}\right\|_{H^{s}}+\left\|u_{0}\right\|_{L^{1}} \leq \delta_{1},
$$

then the global solution delivered by Theorem 1 obeys the estimate

$$
\left\|\partial_{x}^{k} u(t)\right\|_{H^{s-k}} \leq C E_{1}(1+t)^{-1 / 4-k / 2}
$$

for $0 \leq k \leq s-1$, and

$$
\left\|\partial_{x}^{k} q(t)\right\|_{H^{s+1-k}} \leq C E_{1}(1+t)^{-1 / 4-(k+1) / 2}
$$

for $0 \leq k \leq s-2$.

In what follows we work under the assumptions of Theorem 2 .

Also, we mention the following results on the behavior of the global solution, published in [5] and [6]:

$$
\begin{aligned}
\|u(t)\|_{L^{1}} & \leq\left\|u_{0}\right\|_{L^{1}}, \quad\|u(t)\|_{L^{\infty}} \leq\left\|u_{0}\right\|_{L^{\infty}}, \\
\|u(t)\|_{L^{\infty}} & \leq C\left(\frac{\left\|u_{0}\right\|_{L^{1}}}{1+t}\right)^{1 / 2}
\end{aligned}
$$

We say that a function $f$ is exponentially localized if for every $s \geq 0$ there exists a positive constant $C_{s}$ and a number $a_{s}>0$ such that

$$
\left|\partial_{x}^{k} f(x)\right| \leq C_{s} e^{-a_{s}|x|}, \quad x \in R, \quad 0 \leq k \leq s .
$$

In what follows, we restrict ourselves to considering only exponentially localized boundary functions. 
In [7] it was proved that the leading term of the asymptotics for the solution of the initial problem (1.3) coincides with that for the initial problem for the Burgers equation:

$$
\left\{\begin{array}{l}
v_{t}+v \partial_{x} v=\partial_{x}^{2} v, \\
v(x, 0)=u_{0}(x), \quad x \in R
\end{array}\right.
$$

This leading term has the form

$$
u_{0}(x, t)=\frac{2 \zeta_{0}(x, t)}{1-\int_{-\infty}^{x} \zeta_{0}(s, t) d s}
$$

where

$$
\zeta_{0}(x, t)=M_{0}\left(h_{0}\right) \frac{1}{\sqrt{4 \pi t}} e^{-x^{2} / 4 t},
$$

with $h_{0}(x)=(1 / 2) u_{0}(x) \exp \left\{-(1 / 2) \int_{-\infty}^{x} u_{0}(s) d s\right\}$ and $M_{0}\left(h_{0}\right)=\int_{-\infty}^{\infty} h_{0}(s) d s$. In the same paper it was proved that

$$
\left\|\partial_{x}^{k}\left(u(t)-u_{0}(t)\right)\right\|_{L^{2}} \leq C t^{-1 / 4-(k+1) / 2}, \quad k \geq 0,
$$

which implies the estimate

$$
\left\|\partial_{x}^{k}\left(u(t)-u_{0}(t)\right)\right\|_{L^{\infty}} \leq C t^{-1 / 4-k / 2}, \quad k \geq 0 .
$$

In the present paper, we find the second term of the asymptoties for the solution of the Hamer equation; the result looks like this:

$$
u_{1}(x, t)=A\left(u_{0}\right) \frac{1}{\sqrt{t}} \frac{\partial}{\partial x}\left[\frac{2 \zeta_{0}(x, t)}{1-\int_{-\infty}^{x} \zeta_{0}(s, t) d s}\right],
$$

where the factor $A\left(u_{0}\right)$ depends on the initial function $u_{0}$.

\section{§2. Estimates of the solution of Burgers' EQuation}

It is well known that the Burgers equation can be linearized via the Cole-Hopf transformation

$$
v=-2 \frac{\partial_{x} \theta}{\theta}, \quad \theta(x, t)=\exp \left[-\frac{1}{2} \int_{-\infty}^{x} v(s, t) d s\right] .
$$

The function $\theta(x, t)$ solves the heat equation and satisfies the inequality

$$
A=e^{-\frac{1}{2}\left\|u_{0}\right\|_{L_{1}}} \leq \theta(x, t) \leq e^{\frac{1}{2}\left\|u_{0}\right\|_{L^{1}}} .
$$

Instead of $\theta$, we shall consider a function $\zeta, \zeta=-\partial_{x} \theta$, which solves the initial problem

$$
\left\{\begin{array}{l}
\zeta_{t}(x, t)=\partial_{x}^{2} \zeta(x, t) \\
\zeta(x, 0)=(1 / 2) u_{0}(x) \exp \left[-(1 / 2) \int_{-\infty}^{x} u_{0}(s) d s\right]=h_{0}(x)
\end{array}\right.
$$

with exponentially localized initial data. The function $\theta(x, t)$ and the solution $v(x, t)$ of problem (1.6) can be recovered by the solution $\zeta(x, t)$ via the relations

$$
\theta(x, t)=1-\int_{-\infty}^{x} \zeta(s, t) d s, \quad v(x, t)=2 \frac{\zeta(x, t)}{1-\int_{-\infty}^{x} \zeta(s, t)} d s .
$$

The asymptotic expansion of the solution $\zeta$ of problem (2.2) in the powers of $t^{-1 / 2}$ can be obtained from the formula

$$
\zeta(x, t)=\frac{1}{2 \pi} \int_{-\infty}^{\infty} \widehat{h_{0}}(\xi) e^{-\xi^{2} t} e^{i x \xi} d \xi
$$


The Taylor expansion of $\widehat{h_{0}}$ is of the form

$$
\widehat{h_{0}}(\xi)=\sum_{k=0}^{n} \frac{{\widehat{h_{0}}}^{(j)}(0)}{j !} \xi^{j}+\frac{{\widehat{h_{0}}}^{(n+1)}(c)}{(n+1) !} \xi^{n+1},
$$

where $c$ lies between 0 and $\xi$. Substituting this in the above integral, we get

$$
\mathcal{F}[\zeta](\xi, t)=\widehat{h_{0}}(\xi) e^{-\xi^{2} t}=\mathcal{F}\left[\zeta_{n}\right](\xi, t)+\mathcal{F}\left[\gamma_{n}\right](\xi, t) .
$$

Here,

$$
\mathcal{F}\left[\zeta_{n}\right](\xi, t)=\sum_{j=0}^{n} \frac{(-i)^{j} M_{j}\left(h_{0}\right)}{j !} \xi^{j} e^{-\xi^{2} t}
$$

with $M_{j}\left(h_{0}\right)=\int_{-\infty}^{\infty} s^{j} h_{0}(s) d s$, and for $\mathcal{F}\left[\gamma_{n}\right](\xi, t)$ we have

$$
\left|\xi^{k} \mathcal{F}\left[\gamma_{n}\right](\xi, t)\right| \leq C_{n}|\xi|^{n+k+1} e^{-\xi^{2} t} .
$$

We put

$$
v_{n}(x, t)=\frac{2 \zeta_{n}(x, t)}{1-\int_{-\infty}^{x} \zeta_{n}(s, t) d s} .
$$

The next theorem is auxiliary and is well known.

Theorem 3. The solution $v(x, t)$ of equation (1.6) with exponentially localized initial data satisfies the estimate

$$
\left|\partial_{x}^{k}\left(v(x, t)-v_{n}(x, t)\right)\right| \leq C_{n, k} t^{-1-(k+n) / 2}, \quad t \geq 1, \quad k, n=0,1, \ldots,
$$

where $C_{n, k}$ is independent of $x$ and $t$.

\section{§3. Construction of the SeCond term of the Asymptotics FOR THE SOLUTION OF HAMER'S EQUATION}

We return to problem (1.3). The Fourier transform of $K(x)=\frac{1}{2} \exp (-|x|)$ is the function

$$
\widehat{K}(\xi)=\left(1+\xi^{2}\right)^{-1}
$$

Therefore, for the convolution $(K * u)(x, t)=\int_{-\infty}^{\infty} K(x-s) u(s, t) d s$ we have

$$
\widehat{u}-\widehat{K * u}(\xi, t)=\frac{\xi^{2}}{1+\xi^{2}} \widehat{u}(\xi, t) .
$$

We rewrite (1.3) in the form

$$
u_{t}+u \partial_{x} u-\partial_{x}^{2} u=K * \partial_{x}^{2} u-\partial_{x}^{2} u=K * \partial_{x}^{4} u .
$$

Applying the Cole-Hopf transformation $u=-2 \partial_{x} \varphi / \varphi$, we obtain the equation

$$
\left(\frac{\varphi_{t}}{\varphi}\right)_{x}-\left(\frac{\varphi_{x x}}{\varphi}\right)_{x}=K * \partial_{x}^{4} u
$$

Integration with respect to $x$ yields

$$
\begin{aligned}
\varphi_{t}-\partial_{x}^{2} \varphi & =\varphi\left(K * \partial_{x}^{3} u\right)+c(t) \varphi, \\
\varphi(x, 0) & =\exp \left\{-\frac{1}{2} \int_{-\infty}^{x} u_{0}(s) d s\right\}=\varphi_{0}(x) .
\end{aligned}
$$

After multiplication by $\exp \left\{\int c(t) d t\right\}$, the equation takes the form

$$
\Phi_{t}-\Phi_{x x}=\Phi\left(K * \partial_{x}^{3} u\right) \text {, where } \Phi(x, t)=e^{\int c(t) d t} \varphi(x, t) .
$$

Therefore, we assume that $c(t)=0$. 
To make the initial data exponentially localized, we introduce the new function $\psi=$ $-\varphi_{x}$. Problem (3.2) takes the form

$$
\left\{\begin{array}{l}
\psi_{t}-\psi_{x x}=\left[\varphi \partial_{x}^{3}(K * u)\right]_{x} \\
\psi(x, 0)=\frac{1}{2} u_{0}(x) \exp \left\{-\frac{1}{2} \int_{-\infty}^{x} u_{0}(s) d s\right\}=h_{0}(x) .
\end{array}\right.
$$

The solution of the Cauchy problem

$$
\left\{\begin{array}{l}
\psi_{t}=\partial_{x}^{2} \psi+g(x, t) \\
u(x, 0)=h_{0}(x)
\end{array}\right.
$$

can be written via the Poisson formula:

$$
\psi(x, t)=\left(G_{0} * h_{0}\right)(x, t)+\int_{0}^{t}\left(G_{0} * g\right)(x, \tau) d \tau,
$$

where the function

$$
G_{0}(x, t)=\frac{1}{\sqrt{4 \pi t}} e^{-x^{2} / 4 t}=\mathcal{F}^{-1}\left(e^{-\xi^{2} t}\right)(x)
$$

is the fundamental solution of the initial problem for the heat equation. We denote

$$
\zeta(x, t)=\left(G_{0} * h_{0}\right)(x, t)
$$

and

$$
F(\varphi)(x, t)=\int_{0}^{t} G_{0}(t-\tau) *\left[\varphi(\tau)\left(K * \partial_{x}^{3} u\right)(\tau)\right] d \tau .
$$

As in Theorem 3, $\zeta_{0}(x, t)$ will denote the leading term of the asymptotic expansion of the function $\zeta(x, t)$.

The next lemma will serve us for estimating the function $\psi(x, t)$ (see [4]).

Lemma 1. If $\phi \in H^{s}(R) \cap L^{1}(R)$ and $0 \leq k \leq s$, then

$$
\begin{aligned}
& \left\|\partial_{x}^{k} G_{0}(t) * \phi\right\|_{L^{2}}^{2} \leq C(1+t)^{-\frac{1}{2}-k}\|\phi\|_{L^{1}}^{2}+C e^{-2 t}\left\|\partial_{x}^{k} \phi\right\|_{L^{2}}^{2}, \\
& \left\|\partial_{x}^{k} G_{0}(t) * \phi\right\|_{L^{2}}^{2} \leq C t^{-\frac{1}{2}-k}\|\phi\|_{L^{1}}^{2} .
\end{aligned}
$$

Proof. We reproduce the proof of (3.6). We have

$$
\begin{aligned}
& \left\|\partial_{x}^{k} G_{0}(t) * \phi(t)\right\|_{L^{2}}^{2} \leq C\left(\int_{|\xi| \leq 1}+\int_{|\xi|>1}\right)|\xi|^{2 k} e^{-2 \xi^{2} t}|\widehat{\phi}(\xi, t)|^{2} d \xi \\
& \leq C \int_{|\xi| \leq 1} \xi^{2 k} e^{-2 \xi^{2}(t+1)} e^{2 \xi^{2}}|\widehat{\phi}(\xi, t)|^{2} d \xi+C \int_{|\xi|>1} \xi^{2 k} e^{-2 t}|\widehat{\phi}(\xi, t)|^{2} d \xi \\
& \leq C(t+1)^{-1 / 2-k}\|\phi(t)\|_{L^{1}}^{2}+C e^{-2 t}\left\|\partial_{x}^{k} \phi(t)\right\|_{L^{2}}^{2} .
\end{aligned}
$$

Inequality (3.7) is proved in a similar way.

In Lemmas 2 and 3, we estimate the derivatives of the functions $u$ and $\psi$ in the spaces $L^{\infty}$ and $L^{2}$, respectively. We assume that $u_{0} \in H^{s}(R) \cap L^{1}(R)$ with $s \geq 3$.

Lemma 2. For any $k, 0 \leq k \leq s-1$, we have

$$
\left\|\partial_{x}^{k} u(t)\right\|_{L^{\infty}} \leq C(1+t)^{-\frac{k+1}{2}} .
$$


Proof. We represent $\left(\partial_{x}^{k} u(t)\right)^{2}$ as an integral with variable upper limit and apply the Cauchy-Bunyakovskil inequality and estimates of Theorem 2, obtaining

$$
\begin{aligned}
\left\|\partial_{x}^{k} u(t)\right\|_{L^{\infty}} & =\left\|\int_{-\infty}^{x} \partial_{x}\left(\partial_{x}^{k} u(s, t)\right)^{2} d s\right\|_{L^{\infty}}^{1 / 2} \\
& \leq C\left\|\partial_{x}^{k} u(t)\right\|_{L^{2}}^{1 / 2}\left\|\partial_{x}^{k+1} u(t)\right\|_{L^{2}}^{1 / 2} \leq C(1+t)^{-\frac{k+1}{2}} .
\end{aligned}
$$

Lemma 3. For any $k, 0 \leq k \leq s-1$, we have

$$
\left\|\partial_{x}^{k} \psi\right\|_{L^{2}} \leq C(1+t)^{-1 / 4-k / 2} .
$$

Proof. We use induction on $k$. Recall that the functions $\varphi$ and $u$ are related to each other by the Cole-Hopf transformation and that $\psi=-\partial_{x} \varphi$. Therefore,

$$
2 \psi=u \varphi \text {. }
$$

Differentiating this with respect to $x$, we get

$$
2 \partial_{x}^{r} \psi=\varphi \partial_{x}^{r} u+\sum_{k=0}^{r-1} C_{r}^{k} \partial_{x}^{k} u \partial_{x}^{r-k-1} \psi
$$

For $r=1$, Theorem 2 and inequality (1.5) imply

$$
\left\|\partial_{x} \psi\right\|_{L^{2}} \leq C\left(\left\|\partial_{x} u\right\|_{L^{2}}+\|u\|_{L^{\infty}}\|\psi\|_{L^{2}}\right) \leq C(1+t)^{-1 / 4-1 / 2} .
$$

Suppose that for all $k$ with $1 \leq k<r$ we have

$$
\left\|\partial_{x}^{k} \psi\right\|_{L^{2}} \leq C(1+t)^{-1 / 4-k / 2}
$$

Then, by Lemma 2 ,

$$
\left\|\partial_{x}^{r} \psi\right\|_{L^{2}} \leq C\left(\left\|\partial_{x}^{r} u\right\|_{L^{2}}+\sum_{k=0}^{r-1} C_{r}^{k}\left\|\partial_{x}^{k} u\right\|_{L^{\infty}}\left\|\partial_{x}^{r-k-1} \psi\right\|_{L^{2}}\right) \leq C(1+t)^{-1 / 4-r / 2} .
$$

Now we use the fact that the leading term of the asymptotics for problem (1.3) is known.

Lemma 4. The function $x u(x, t)$ is integrable with respect to $x$ over $\mathbb{R}$, and

$$
\int_{-\infty}^{\infty}|x u(x, t)| d x \leq C(1+t)^{\frac{1}{2}}
$$

Proof. We write the solution $u(x, t)$ of Hamer's equation in the form

$$
u(x, t)=\left(u-u_{0}\right)(x, t)+u_{0}(x, t),
$$

where $u_{0}(x, t)$ is the leading term of the asymptotics for the solution of problem (1.3). It is easily seen that

$$
\int_{-\infty}^{\infty}\left|x u_{0}(x, t)\right| d x \leq C \sqrt{t}
$$

Putting $w(x, t)=u(x, t)-u_{0}(x, t)$, we have

$$
w w_{x}=u \partial_{x} u+u_{0} \partial_{x} u_{0}-\partial_{x}\left(u_{0} w\right) .
$$

Since $u_{0}(x, t)$ satisfies the Burgers equation

$$
u_{0 t}+u_{0} u_{0 x}=\partial_{x}^{2} u_{0},
$$

we can subtract (3.1) from (3.10) to get

$$
w_{t}+w w_{x}=(K * w-w)+K * \partial_{x}^{4} u_{0}-\partial_{x}\left(u_{0} w\right) .
$$


We employ the inequality

$$
|w|_{t}+\frac{1}{2}\left(w^{2} \operatorname{sgn}(w)\right)_{x} \leq(K *|w|-|w|)+\left(K *\left(\partial_{x}^{4} u_{0}\right)\right) \operatorname{sgn}(w)-\partial_{x}\left(u_{0} w\right) \operatorname{sgn}(w)
$$

(see [8]), which is valid a.e. Put $L(v)=(K * v)-v$. We multiply the inequality above by $|x|$ and integrate in $x$ :

$$
\begin{aligned}
& \frac{d}{d t} \int_{-\infty}^{\infty}|x w(x, t)| d x \leq \int_{-\infty}^{\infty}|w(x, t)| L(|x|) d x \\
& \quad+\frac{1}{2} \int_{-\infty}^{\infty} w^{2}(x, t) \operatorname{sgn}(x w(x, t)) d x+\int_{-\infty}^{\infty}\left(K * \partial_{x}^{4} u_{0}\right)(x, t)|x| \operatorname{sgn}(w(x, t)) d s \\
& \quad-\int_{-\infty}^{\infty} \partial_{x}\left(u_{0}(x, t) w(x, t)\right)|x| \operatorname{sgn}(w(x, t)) d x
\end{aligned}
$$

Observe that $L(|x|)=e^{-|x|}$. Integrating by parts in the last two integrals and applying the Cauchy-Bunyakovskiu inequality, we obtain the estimate

$$
\frac{d}{d t} \int_{-\infty}^{\infty}|x w(x, t)| d x \leq C\left(\|w\|_{L^{2}}+\|w\|_{L^{2}}^{2}+\left\|\partial_{x}^{3} u_{0}\right\|_{L^{1}}+\left\|u_{0}\right\|_{L^{2}}\|w\|_{L^{2}}\right) .
$$

Since

$$
\left.\|w(t)\|_{L^{2}} \leq C t^{-3 / 4} \quad(\text { see }[7]) \quad \text { and } \quad \| \partial_{x}^{3} u_{0}(t)\right) \|_{L^{1}} \leq C t^{-3 / 2}
$$

we have

$$
\frac{d}{d t} \int_{-\infty}^{\infty}|x w(x, t)| d x \leq C t^{-3 / 4}, \quad t>1
$$

Therefore,

$$
\int_{-\infty}^{\infty}|x w(x, t)| d x \leq \int_{-\infty}^{\infty}|x w(x, 1)| d x+C t^{1 / 4}, \quad t>1 .
$$

Combining this and (3.9), we get the required claim.

Now we state our main theorem.

Theorem 4. Suppose that, as in Theorem 2, $u_{0}(x) \in H^{s}(R) \cap L^{1}(R)$ with $s \geq 5$, and that this function is exponentially localized. Let $u(x, t)$ be the solution of the initial problem (1.3). Then

$$
\left|\partial_{x}^{k}\left(u(x, t)-u_{0}(x, t)-u_{1}(x, t)\right)\right| \leq C t^{-\frac{k+3}{2}} \log (1+t), \quad t>1,
$$

for all $k$ with $0 \leq k<s-4$, with a constant $C$ independent of $x$ and $t$. Here $u_{0}(x, t)$ and $u_{1}(x, t)$ are the functions (1.7) and (1.11), respectively.

Proof. We start with estimating the function $F(\varphi)(x, t)$ (see (3.5)), then find the second asymptotic term for the solution of Hamer's equation, and finally, prove estimate (3.11).

I. We write $F(\varphi)(x, t)$ as a sum:

$$
\begin{aligned}
\partial_{x} \int_{0}^{t} d \tau \int_{-\infty}^{\infty} G_{0}(x-\xi, t-\tau)\left[\varphi(\xi, \tau)\left(K * \partial_{\xi}^{2} u\right)(\xi, \tau)\right] d \xi \\
\quad-\int_{0}^{t} d \tau \int_{-\infty}^{\infty} G_{0}(x-\xi, t-\tau)\left[\psi(\xi, \tau)\left(K * \partial_{\xi}^{2} u\right)(\xi, \tau)\right] d \xi \\
=\partial_{x} F_{1}(\varphi)(x, t)+F_{2}(\psi)(x, t)
\end{aligned}
$$


For $m \geq 2$, the $L^{2}$-norm of $\partial_{x}^{m} F_{1}(\varphi)$ is dominated by

$$
\begin{aligned}
\int_{0}^{t / 2} & \left\|G_{0}(\tau) * \partial_{x}^{m}\left[\varphi(t-\tau)\left(K * \partial_{x}^{2} u\right)(t-\tau)\right]\right\|_{L^{2}} d \tau \\
& +\int_{0}^{t / 2}\left\|\partial_{x}^{m} G_{0}(t-\tau) *\left[\varphi(\tau)\left(K * \partial_{x}^{2} u\right)(\tau)\right]\right\|_{L^{2}} d \tau=I_{1}+I_{2} .
\end{aligned}
$$

We estimate $I_{1}$, applying Lemmas $1-3$ and estimate (1.4):

$$
\begin{aligned}
I_{1} \leq C & {\left[\int_{0}^{t / 2}\left\|G_{0}(\tau) *\left[\varphi(t-\tau) \partial_{x}^{m+2}(K * u)(t-\tau)\right]\right\|_{L^{2}} d \tau\right.} \\
& \left.+\sum_{k=1}^{m} C_{m}^{k} \int_{0}^{t / 2} \tau^{-\frac{1}{4}}\left\|\partial_{x}^{k} \varphi(t-\tau) \partial_{x}^{m+2-k}(K * u)(t-\tau)\right\|_{L^{1}} d \tau\right] \\
\leq C & \int_{0}^{t / 2}\left\|\left(K * \partial_{x}^{m+2} u\right)(t-\tau)\right\|_{L^{2}} d \tau \\
& \quad+C \sum_{k=1}^{m} \int_{0}^{t / 2} \tau^{-\frac{1}{4}}\left\|\partial_{x}^{k} \varphi(t-\tau)\right\|_{L^{2}}\left\|\partial_{x}^{m+2-k}(K * u)(t-\tau)\right\|_{L^{2}} d \tau \\
\leq C & (1+t)^{-1 / 4-m / 2} .
\end{aligned}
$$

Now we treat the second term $I_{2}$ in (3.13). Integrating by parts and using (1.4) and Lemma 1-3, we get

$$
\begin{aligned}
I_{2} \leq C & \sum_{k=0}^{2} \int_{0}^{t / 2}\left\|\partial_{x}^{m+k} G_{0}(t-\tau) *\left[\partial_{x}^{2-k} \varphi(\tau)(K * u)(\tau)\right]\right\|_{L^{2}} \\
\leq C & {\left[\sum_{k=0}^{2}(1+t)^{-1 / 4-(m+k) / 2} \int_{0}^{t / 2}\left\|\partial_{x}^{2-k} \varphi(\tau)(K * u)(\tau)\right\|_{L^{1}} d \tau\right.} \\
& \left.+\sum_{k=0}^{2} e^{-t / 2} \int_{0}^{t / 2}\left\|\partial_{x}^{m+k}\left[\partial_{x}^{2-k} \varphi(\tau)(K * u)(\tau)\right]\right\|_{L^{2}} d \tau\right] \\
\leq C & {\left[(1+t)^{-1 / 4-(m+2) / 2} \int_{0}^{t / 2}\|(K * u)(\tau)\|_{L^{1}} d \tau\right.} \\
& +\sum_{k=0}^{1}(1+t)^{-1 / 4-(m+k) / 2} \int_{0}^{t / 2}\left\|\partial_{x}^{2-k} \varphi(\tau)\right\|_{L^{2}}\|(K * u)(\tau)\|_{L^{2}} d \tau \\
& \left.+e^{-t / 2} \int_{0}^{t / 2}(1+\tau)^{-(m+2) / 2}\right] \leq C(1+t)^{-1 / 4-m / 2} \log (1+t)
\end{aligned}
$$

Relations (3.13), (3.14), and (3.15) imply the inequality

$$
\left\|\partial_{x}^{m} F_{1}(\varphi)\right\|_{L^{2}} \leq C(1+t)^{-\frac{1}{4}-\frac{m}{2}} \log (1+t), \quad m=2,3, \ldots
$$

Denoting

$$
p(\xi, \tau)=\psi(\xi, \tau)\left(K * \partial_{\xi}^{2} u\right)(\xi, \tau)
$$

we put

$$
F_{0}(\psi)(x, t)=G_{0}(x, t) \int_{0}^{t} \int_{-\infty}^{\infty} p(\mu, \tau) d \mu d \tau
$$


and represent the function $F_{3}(\psi)(x, t)=F_{2}(\psi)(x, t)-F_{0}(\psi)(x, t)$ (see (3.12) $)$ in the form

$$
\begin{gathered}
\int_{0}^{t / 2} \int_{-\infty}^{\infty} G_{0}(\xi, \tau) p(x-\xi, t-\tau) d \xi d \tau-G_{0}(x, t) \int_{0}^{t / 2} \int_{-\infty}^{\infty} p(\mu, t-\tau) d \mu d \tau \\
\quad+\int_{0}^{t / 2} d \tau \int_{-\infty}^{\infty}\left[G_{0}(x-\xi, t-\tau)-G_{0}(x, t)\right] p(\xi, \tau) d \xi \\
=J_{1}(x, t)+J_{2}(x, t)+J_{3}(x, t) .
\end{gathered}
$$

Suppose $m \geq 1$. To estimate the $L^{2}$-norm of $\partial_{x}^{m} F_{3}(\psi)(x, t)$, we start with applying Lemma 1 to $\partial_{x}^{m} J_{1}(x, t)$ :

$$
\left\|\partial_{x}^{m} J_{1}(t)\right\|_{L^{2}} \leq C \int_{0}^{t / 2} \tau^{-\frac{1}{4}}\left\|\partial_{x}^{m}\left[\psi(t-\tau) \partial_{x}^{2}(K * u)(t-\tau)\right]\right\|_{L^{1}} d \tau
$$

Since

$$
\begin{aligned}
\left\|\partial_{x}^{m}\left[\psi(t-\tau) \partial_{x}^{2}(K * u)(t-\tau)\right]\right\|_{L^{1}} & \leq C \sum_{k=0}^{m}\left\|\partial_{x}^{k} \psi(t-\tau)\right\|_{L^{2}}\left\|\partial_{x}^{m+2-k}(K * u)(t-\tau)\right\|_{L^{2}} \\
& \leq C(1+t)^{-\frac{m+1}{2}-1},
\end{aligned}
$$

we see that

$$
\int_{0}^{t / 2} \tau^{-\frac{1}{4}}\left\|\partial_{x}^{m}\left[\psi(t-\tau) \partial_{x}^{2}(K * u)(t-\tau)\right]\right\|_{L^{1}} d \tau \leq C t^{-\frac{1}{4}-\frac{m+1}{2}}
$$

Thus, we arrive at the inequality

$$
\left\|\partial_{x}^{m} J_{1}(t)(\psi)\right\|_{L^{2}} \leq C t^{-\frac{1}{4}-\frac{m+1}{2}}, \quad t>1, m \geq 1 .
$$

We estimate $J_{2}(x, t)$. Since

$$
\left\|\partial_{x}^{m} G_{0}(t-\tau)\right\|_{L^{2}} \leq C(t-\tau)^{-\frac{m}{2}-\frac{1}{4}} \text { and } \int_{-\infty}^{\infty}|p(\xi, t-\tau)| d \xi \leq C(t-\tau)^{-\frac{3}{2}}
$$

it follows that the second summand on the right in (3.17) satisfies the estimate

$$
\left\|\partial_{x}^{m} G_{0}(t-\tau)\right\|_{L^{2}} \int_{0}^{t / 2} \int_{-\infty}^{\infty}|p(\mu, t-\tau)| d \mu d \tau \leq C t^{-\frac{1}{4}-\frac{m+1}{2}}, \quad t>1,
$$

which is proved with the help of (1.4).

Passing to $J_{3}(x, t)$, we write the difference $G_{0}(x-\xi, t-\tau)-G_{0}(x, t)$ in the form

$$
\int_{0}^{1} \frac{d}{d \nu} G_{0}(x-\xi \nu, t-\tau \nu) d \nu=\int_{0}^{1}\left[-\xi \partial_{x} G_{0}(x-\xi \nu, t-\tau \nu)-\tau \partial_{t} G_{0}(x-\xi \nu, t-\tau \nu)\right] d \nu
$$

By Lemmas 2 and 4 , we have

$$
\begin{array}{rl}
\int_{0}^{t / 2} & d \tau \int_{-\infty}^{\infty}\left|\xi \psi(\xi, \tau)\left(K * \partial_{\xi}^{2} u\right)(\xi, \tau)\right| d \xi \\
\quad \leq \int_{0}^{t / 2}(1+\tau)^{-\frac{3}{2}}\|\xi u(\xi, \tau)\|_{L^{1}} d \tau \leq \int_{0}^{t / 2}(1+\tau)^{-1} d \tau \leq C \log (1+t) .
\end{array}
$$


Then, by Lemma 1 .

$$
\begin{aligned}
\left\|\int_{0}^{t / 2} d \tau \int_{0}^{1} d \nu \int_{-\infty}^{\infty} \partial_{x}^{m+1} G_{0}(x-\xi \nu, t-\tau \nu) \xi p(\xi, \tau) d \xi\right\|_{L^{2}} \\
\quad \leq \int_{0}^{t / 2} d \tau \int_{0}^{1} d \nu\left\|\int_{-\infty}^{\infty} \partial_{x}^{m+1} G_{0}(x-\xi \nu, t-\tau \nu) \xi p(\xi, \tau) d \xi\right\|_{L^{2}} \\
\quad \leq C \int_{0}^{t / 2} d \tau \int_{0}^{1} d \nu(t-\tau \nu)^{-\frac{1}{4}-\frac{m+1}{2}}\|\xi p(\xi, \tau)\|_{L^{1}} d \tau \\
\quad \leq C t^{-\frac{1}{4}-\frac{m+1}{2}} \int_{0}^{t / 2}\|\xi p(\xi, \tau)\|_{L^{1}} \leq C t^{-\frac{1}{4}-\frac{m+1}{2}} \log (1+t), \quad t>1 .
\end{aligned}
$$

Next we apply Lemma 2 to obtain the inequality

$$
\begin{aligned}
\| \int_{0}^{t / 2} & \tau d \tau \int_{0}^{1} d \nu \int_{-\infty}^{\infty} \partial_{x}^{m} \partial_{t} G_{0}(x-\xi \nu, t-\tau \nu) p(\xi, \tau) d \xi \|_{L^{2}} \\
& \leq\left\|\int_{0}^{t / 2} \tau d \tau \int_{0}^{1} d \nu \int_{-\infty}^{\infty} \partial_{x}^{m+2} G_{0}(x-\xi \nu, t-\tau \nu) p(\xi, \tau) d \xi\right\|_{L^{2}} \\
& \leq \int_{0}^{t / 2} \tau d \tau \int_{0}^{1} d \nu\left\|\int_{-\infty}^{\infty} \partial_{x}^{m+2} G_{0}(x-\xi \nu, t-\tau \nu) p(\xi, \tau) d \xi\right\|_{L^{2}} \\
& \leq C \int_{0}^{t / 2} \tau d \tau \int_{0}^{1} d \nu(t-\tau \nu)^{-\frac{1}{4}-\frac{m+2}{2}}\|p(\xi, \tau)\|_{L^{1}} d \tau \\
& \leq C t^{-\frac{1}{4}-\frac{m+2}{2}} \int_{0}^{t / 2} \tau(1+\tau)^{-\frac{3}{2}} d \tau \leq C t^{-\frac{1}{4}-\frac{m+1}{2}}, \quad t>1 .
\end{aligned}
$$

As a result, for $t>1$ we get an estimate for $\partial_{x}^{m} F_{3}(\psi), m \geq 1$ :

$$
\left\|\partial_{x}^{m} F_{3}(\psi)\right\|_{L^{2}} \leq \sum_{k=1}^{3}\left\|\partial_{x}^{m} J_{k}(t)\right\|_{L^{2}} \leq C t^{-\frac{1}{4}-\frac{m+1}{2}} \log (1+t) .
$$

Thus, the function $F(\varphi)$ (see (3.5)) has the form

$$
\partial_{x} F_{1}(\varphi)(x, t)+F_{3}(\psi)(x, t)+F_{0}(\psi)(x, t),
$$

and for $t>1$ we have

$$
\left\|\partial_{x}^{m+1} F_{1}(\varphi)\right\|_{L^{2}}+\left\|\partial_{x}^{m} F_{3}(\psi)\right\|_{L^{2}} \leq t^{-\frac{1}{4}-\frac{m+1}{2}} \log (1+t), \quad m \geq 1 .
$$

II. The solutions of the initial Hamer problem and of problem (3.3) are related to each other by the formula

$$
u(x, t)=2 \frac{\psi(x, t)}{1-\int_{\infty}^{x} \psi(s, t) d s} .
$$

We write the formal asymptotic expansion of the solution of (3.3):

$$
\psi(x, t)=\sum_{k=0}^{\infty} \psi_{k}(y)\left(\frac{1}{\sqrt{t}}\right)^{k+1}, \quad y=\frac{x}{\sqrt{t}} .
$$


Substituting this in $(3.20)$ yields

$$
\begin{aligned}
u(x, t) & =2 \frac{\sum_{k=0}^{\infty} \psi_{k}(y)\left(\frac{1}{\sqrt{t}}\right)^{k+1}}{1-\int_{-\infty}^{y} \psi_{0}(s) d s-\sum_{k=1}^{\infty}\left(\frac{1}{\sqrt{t}}\right)^{k} \int_{-\infty}^{y} \psi_{k}(s) d s} \\
& =2 \frac{\psi_{0}(y)}{1-\int_{-\infty}^{y} \psi_{0}(s) d s} \frac{1}{\sqrt{t}}+2 \frac{\psi_{1}(y)}{1-\int_{-\infty}^{y} \psi_{0}(s) d s} \frac{1}{t}+2 \frac{\psi_{0}(y) \int_{-\infty}^{y} \psi_{1}(s) d s}{\left(1-\int_{-\infty}^{y} \psi_{0}(s) d s\right)^{2}} \frac{1}{t}+\ldots \\
= & 2 \frac{\psi_{0}(y)}{1-\int_{-\infty}^{y} \psi_{1}(s) d s} \frac{1}{\sqrt{t}}+2\left(\frac{\psi_{0}(y) \int_{-\infty}^{y} \psi_{1}(s) d s+\psi_{1}(y)\left(1-\int_{-\infty}^{y} \psi_{0}(s) d s\right)}{\left(1-\int_{-\infty}^{y} \psi_{0}(s) d s\right)^{2}}\right) \frac{1}{t}+\ldots
\end{aligned}
$$

To avoid introducing new notation, we assume that

$$
u(x, t)=\sum_{k=0}^{\infty} u_{k}\left(\frac{x}{\sqrt{t}}\right)\left(\frac{1}{\sqrt{t}}\right)^{k+1} \text { and } u(y, t)=\sum_{k=0}^{\infty} u_{k}(y)\left(\frac{1}{\sqrt{t}}\right)^{k+1} .
$$

Comparing (3.21) and (3.22), we see that

$$
u_{0}(y)=2 \frac{\psi_{0}(y)}{1-\int_{-\infty}^{y} \psi_{0}(s) d s} \text { and } u_{1}(y)=2 \frac{d}{d y}\left[\frac{\int_{-\infty}^{y} \psi_{1}(s) d s}{1-\int_{-\infty}^{y} \psi_{0}(s) d s}\right] .
$$

In the automodel variables $(y, t)$, the second term of the formal asymptotics for the solution of Hamer's equation satisfies

$$
-v(y)-\frac{1}{2} y v_{y}(y)+v(y) u_{0 y}(y)+u_{0}(y) v_{y}(y)=v_{y y}(y)
$$

A direct calculation shows that the function $\widetilde{u}_{1}(y)=\frac{\partial}{\partial y} u_{0}(y)$ is a solution of equation (3.24).

Another linearly independent solution of (3.24) can be sought in the form $v(y)=$ $\widetilde{u}_{1}(y) w(y)$. Plugging $v(y)$ in (3.24) and putting $w_{y}=\varkappa$, we arrive at the equation

$$
\left(-\frac{1}{2} y \widetilde{u}_{1}(y)+u_{0}(y) \widetilde{u}_{1}(y)-2 \widetilde{u}_{1 y}(y)\right) \varkappa(y)=\widetilde{u}_{1}(y) \varkappa_{y}(y) .
$$

The following function solves (3.25) (up to a factor):

$$
\varkappa(y)=e^{-\frac{y^{2}}{4}} \frac{1}{\widetilde{u}_{1}^{2}(y)} e^{\int u_{0}(y) d y} .
$$

Since

where

$$
u_{0}(y)=\frac{2 A_{0} \frac{1}{\sqrt{4 \pi}} e^{-\frac{y^{2}}{4}}}{1-A_{0} \Phi_{0}\left(\frac{y}{\sqrt{2}}\right)}
$$

$$
A_{0}=\frac{M_{0}\left(h_{0}\right)}{1-\frac{1}{2} M_{0}\left(h_{0}\right)}, \text { and } \Phi_{0}(y) \text { is the Laplace function, }
$$

we have

$$
\int_{-\infty}^{y} u_{0}(s) d s=-2 \log \left(1-A_{0} \Phi_{0}\left(\frac{y}{\sqrt{2}}\right)\right)
$$

Finally,

Since

$$
\varkappa(y)=e^{-\frac{y^{2}}{4}} \frac{1}{\widetilde{u}_{1}^{2}(y)}\left(1-A_{0} \Phi_{0}\left(\frac{y}{\sqrt{2}}\right)\right)^{-2} .
$$

$$
\widetilde{u}_{1}(y) \int_{0}^{y} e^{-s^{2} / 4}\left(\widetilde{u}_{1}(s)\right)^{-2}\left(1-A_{0} \Phi_{0}(s / \sqrt{2})\right)^{-2} d s \sim \frac{c}{y}, \quad|y| \rightarrow \infty,
$$


the second linearly independent solution of (3.24) behaves at infinity as a power function. Thus, up to a factor, the exponentially localized formal solution of equation (3.24) looks like this:

$$
\widetilde{u}_{1}(y)=2 \frac{d}{d y}\left[\frac{\psi_{0}(y)}{1-\int_{-\infty}^{y} \psi_{0}(s) d s}\right] .
$$

Comparing of (3.23) with (3.26), we see that

$$
\varphi_{0}(y)\left(\partial_{y} \psi_{0}(y)-\psi_{1}(y)\right)+\psi_{0}(y)\left(\psi_{0}(y)-\int_{-\infty}^{y} \psi_{1}(s) d s\right)=0 .
$$

Put $\chi(y)=\psi_{0}(y)-\int_{-\infty}^{y} \psi_{1}(s) d s$. Since $\psi_{0}(y)=-\partial_{y} \varphi_{0}(y)$, we have

$$
\partial_{y}\left(\chi / \varphi_{0}\right)(y)=0, \quad y \in(-\infty, \infty) .
$$

so that the function $\chi / \varphi_{0}$ is a constant. Since $\left(\chi / \varphi_{0}\right)(-\infty)=0$, it follows that $\psi_{0}(y)=$ $\int_{-\infty}^{y} \psi_{1}(s) d s$. Hence, $u_{1}(y)$ in (3.23) coincides with $\widetilde{u}_{1}(y)$ in (3.26).

III. Recall that the leading term $\psi_{0}(x, t)$ of the solution $\psi(x, t)$ of problem (3.3) coincides with the function $\zeta_{0}(x, t)$ (see (1.8)).

We write $\psi(x, t)$ (see (2.3), (2.4), and (3.18)) in the form

$$
\begin{aligned}
\psi(x, t) & =\zeta(x, t)+\partial_{x} F(\varphi)(x, t) \\
& =\zeta_{0}(x, t)+\gamma_{0}(x, t)+\partial_{x}^{2} F_{1}(\varphi)(x, t)+\partial_{x} F_{3}(\psi)(x, t)+\partial_{x} F_{0}(\psi) \\
& =\psi_{0}(x, t)+\psi_{1}(x, t)+\delta_{1}(x, t),
\end{aligned}
$$

where

$$
\psi_{1}(x, t)=\left(-M_{1}\left(h_{0}\right)+\int_{0}^{\infty} \int_{-\infty}^{\infty} p(\mu, \tau) d \mu d \tau\right) \partial_{x} G_{0}(x, t)
$$

and $\delta_{1}(x, t)$ has the form

$$
\partial_{x}^{2} F_{1}(\varphi)(x, t)+\partial_{x} F_{3}(\psi)(x, t)+\partial_{x} G_{0}(x, t) \int_{t}^{\infty} \int_{-\infty}^{\infty} p(\mu, t) d \mu d \tau
$$

(see (2.5) and (3.19) ) and satisfies the estimates

$$
\begin{aligned}
\left\|\partial_{x}^{m} \delta_{1}(x, t)\right\|_{L^{2}} & \leq C t^{-\frac{1}{4}-\frac{m+2}{2}} \log (1+t), \quad m \geq 0, \\
\left\|\int_{-\infty}^{x} \delta_{1}(x, t)\right\|_{L^{2}} & \leq C t^{-\frac{3}{4}} \log (1+t) .
\end{aligned}
$$

We represent the difference $u(x, t)-u_{0}(x, t)$ with $u_{0}(x, t)$ as in (1.7) in the form

$$
\begin{aligned}
u(x, t)- & u_{0}(x, t) \\
= & 2 \frac{\psi_{0}(t, x) \int_{-\infty}^{x} \delta_{0}(s, t) d s+\delta_{0}(x, t)\left(1-\int_{-\infty}^{x} \psi_{0}(s, t) d s\right)}{\varphi(x, t)\left(1-\int_{-\infty}^{x} \psi_{0}(s, t) d s\right)} \\
= & 2 \frac{\psi_{0}(x, t) \int_{-\infty}^{x} \psi_{1}(s, t) d s+\psi_{1}(x, t)\left(1-\int_{-\infty}^{x} \psi_{0}(s, t) d s\right)}{\varphi(x, t)\left(1-\int_{-\infty}^{x} \psi_{0}(s, t) d s\right)} \\
& +2 \frac{\psi_{0}(x, t) \int_{-\infty}^{x} \delta_{1}(s, t) d s+\delta_{1}(x, t)\left(1-\int_{-\infty}^{x} \psi_{0}(s, t) d s\right)}{\varphi(x, t)\left(1-\int_{-\infty}^{x} \psi_{0}(s, t) d s\right)} \\
=2 & \frac{\partial}{\partial x}\left[\frac{\int_{-\infty}^{x} \psi_{1}(s, t) d s}{\left(1-\int_{-\infty}^{x} \psi_{0}(s, t) d s\right)}\right] \frac{1-\int_{-\infty}^{x} \psi_{0}(s, t) d s}{\varphi(x, t)} \\
& +2 \frac{\partial}{\partial x}\left[\frac{\int_{-\infty}^{x} \delta_{1}(s, t) d s}{\left(1-\int_{-\infty}^{x} \psi_{0}(s, t) d s\right)}\right] \frac{1-\int_{-\infty}^{x} \psi_{0}(s, t) d s}{\varphi(x, t)} .
\end{aligned}
$$


Here $\delta_{0}(x, t)=\psi(x, t)-\psi_{0}(x, t)$. Put

$$
u_{1}(x, t)=2 \frac{\partial}{\partial x}\left[\frac{\int_{-\infty}^{x} \psi_{1}(s, t) d s}{\left(1-\int_{-\infty}^{x} \psi_{0}(s, t) d s\right)}\right],
$$

where $\psi_{1}(x, t)$ is as in (3.27). Then

$$
\begin{aligned}
u(x, t)- & u_{0}(x, t)-u_{1}(x, t) \\
=2 & \frac{\partial}{\partial x}\left[\frac{\int_{-\infty}^{x} \delta_{1}(s, t) d s}{\left(1-\int_{-\infty}^{x} \psi_{0}(s, t) d s\right)}\right] \frac{1-\int_{-\infty}^{x} \psi_{0}(s, t) d s}{\varphi(x, t)} \\
& +2 \frac{\partial}{\partial x}\left[\frac{\int_{-\infty}^{x} \psi_{1}(s, t) d s}{\left(1-\int_{-\infty}^{x} \psi_{0}(s, t) d s\right)}\right] \frac{\int_{-\infty}^{x} \delta_{0}(s, t) d s}{\varphi(x, t)}=J_{1}(x, t)+J_{2}(x, t) .
\end{aligned}
$$

Since

$$
\int_{-\infty}^{x} \psi_{0}(s, t) d s=M_{0}\left(h_{0}\right) \int_{-\infty}^{x} \frac{1}{\sqrt{4 \pi t}} e^{-\frac{s^{2}}{4 t}} d s \leq M_{0}\left(h_{0}\right)
$$

and

$$
M_{0}\left(h_{0}\right)=1-e^{-\frac{1}{2} M_{0}\left(u_{0}\right)} \quad(\text { see }(3.3))
$$

we have

$$
1-\int_{-\infty}^{x} \psi_{0}(s, t) d s \geq 1-M_{0}\left(h_{0}\right)=e^{-\frac{1}{2} M_{0}\left(u_{0}\right)}>0 .
$$

Combining this with (2.1), we see that the denominator in (3.30) is bounded from below.

To estimate the function $\partial_{x}^{k}\left(u(t)-u_{0}(t)-u_{1}(t)\right)$ in $L^{2}(R)$, we start with considering the derivative of the first summand $J_{1}(x, t)$ in (3.30). Estimates (3.28) and (3.29) obtained earlier allow us to conclude that

$$
\begin{aligned}
& \left\|\partial_{x}^{k}\left[\psi_{0}(x, t) \int_{-\infty}^{x} \delta_{1}(s, t) d s\right]\right\|_{L^{2}} \leq C \sum_{j=0}^{k}\left\|\partial_{x}^{j} \psi_{0}(x, t) \partial_{x}^{k-j} \int_{-\infty}^{x} \delta_{1}(s, t) d s\right\|_{L^{2}} \\
& \quad \leq \sum_{j=0}^{k}\left\|\partial_{x}^{j} \psi_{0}(x, t)\right\|_{L^{\infty}}\left\|\partial_{x}^{k-j} \int_{-\infty}^{x} \delta_{1}(s, t) d s\right\|_{L^{2}} \leq C \sum_{j=0}^{k} t^{-\frac{1}{2}-\frac{j}{2}} t^{-\frac{1}{4}-\frac{k-j+1}{2}} \log (1+t) \\
& \quad=C t^{-\frac{1}{4}-\frac{k+2}{2}} \log (1+t)
\end{aligned}
$$

and

$$
\begin{aligned}
& \left\|\partial_{x}^{k}\left[\delta_{1}(x, t)\left(1-\int_{-\infty}^{x} \psi_{0}(s, t) d s\right)\right]\right\|_{L^{2}} \\
& \quad \leq C \sum_{j=0}^{k}\left\|\partial_{x}^{j} \delta_{1}(x, t) \partial_{x}^{k-j}\left(1-\int_{-\infty}^{x} \psi_{0}(s, t) d s\right)\right\|_{L^{2}} \\
& \quad \leq C t^{-\frac{1}{4}-\frac{k+2}{2}} \log (1+t)+\sum_{j=0}^{k-1}\left\|\partial_{x}^{j} \delta_{1}(x, t)\right\|_{L^{2}}\left\|\partial_{x}^{k-j} \int_{-\infty}^{x} \psi_{0}(s, t) d s\right\|_{L^{\infty}} \\
& \leq C \sum_{j=0}^{k} t^{-\frac{1}{4}-\frac{j+2}{2}} \log (1+t) t^{-\frac{k-j}{2}} \leq C t^{-\frac{1}{4}-\frac{k+2}{2}} \log (1+t) .
\end{aligned}
$$

Thus,

$$
\begin{aligned}
\| \partial_{x}^{k}\left[\delta_{1}(x, t)\left(1-\int_{-\infty}^{x} \psi_{0}(s, t) d s\right)+\psi_{0}(x, t) \int_{-\infty}^{x}\right. & \left.\delta_{1}(s, t) d s\right] \|_{L^{2}} \\
& \leq C t^{-\frac{1}{4}-\frac{k+2}{2}} \log (1+t) .
\end{aligned}
$$


The first summand $J_{1}(x, t)$ in (3.30) can be written as $\mu(x, t) / \nu(x, t)$, where $\mu(x, t)$ is the expression in the square brackets in (3.31), and $\nu(x, t)$ is the product of $\varphi(x, t)$ and $\left(1-\int_{-\infty}^{x} \psi_{0}(s, t) d s\right)$. Differentiating the relation

$$
\mu(x, t)=J_{1}(x, t) \nu(x, t)
$$

with respect to $x$, we get

$$
\begin{aligned}
& \left\|\partial_{x}^{k} J_{1}(x, t)\right\|_{L^{2}} \\
& \quad \leq\left[\left\|\partial_{x}^{k} \mu(x, t)\right\|_{L^{2}}+\sum_{j=0}^{k-1}\left\|\partial_{x}^{j} J_{1}(x, t)\right\|_{L^{2}}\left\|\partial_{x}^{k-j} \nu(x, t)\right\|_{L^{\infty}}\right]\|1 / \nu(x, t)\|_{L^{\infty}} .
\end{aligned}
$$

In (3.32), for all $j=0, \ldots, k-1$ we have

$$
\left\|\partial_{x}^{k-j} \nu(x, t)\right\|_{L^{\infty}} \leq C t^{\frac{k-j}{2}}
$$

and

Assuming that

$$
\left\|\partial_{x}^{j} \mu(x, t)\right\|_{L^{2}} \leq C t^{-\frac{1}{4}-\frac{j+2}{2}} \log (1+t) .
$$

$$
\left\|\partial_{x}^{j} J_{1}(x, t)\right\|_{L^{2}} \leq C t^{-\frac{1}{4}-\frac{j+2}{2}} \log (1+t), \quad j=0, \ldots, k-1,
$$

for $j=k$ we arrive at the inequality

$$
\left\|\partial_{x}^{k} J_{1}(x, t)\right\|_{L^{2}} \leq C t^{-\frac{1}{4}-\frac{k+2}{2}} \log (1+t), \quad t \geq 1 .
$$

The second summand $J_{2}(x, t)$ can be written in the form of the product of

$$
\frac{\psi_{1}(x, t)\left(1-\int_{-\infty}^{x} \psi_{0}(s, t) d s\right)+\psi_{0}(x, t) \int_{-\infty}^{x} \psi_{1}(s, t) d s}{\left(1-\int_{-\infty}^{x} \psi_{0}(s, t) d s\right) \varphi(x, t)}=J_{21}(x, t)
$$

and

$$
\frac{\int_{-\infty}^{x} \delta_{0}(s, t) d s}{\left(1-\int_{-\infty}^{x} \psi_{0}(s, t) d s\right)}=J_{22}(x, t) .
$$

The derivative of the first summand in the numerator of the ratio $J_{21}(x, t)$ has the form

$$
\begin{aligned}
& \partial_{x}^{k}\left(\psi_{1}(x, t)\left(1-\int_{-\infty}^{x} \psi_{0}(s, t) d s\right)\right) \\
& \quad=\left(1-\int_{-\infty}^{x} \psi_{0}(s, t) d s\right) \partial_{x}^{k} \psi_{1}(x, t)-\sum_{j=0}^{k-1} \partial_{x}^{j} \psi_{1}(x, t) \partial_{x}^{k-j-1} \psi_{0}(x, t) .
\end{aligned}
$$

Therefore,

$$
\begin{aligned}
& \left\|\partial_{x}^{k}\left[\psi_{1}(x, t)\left(1-\int_{-\infty}^{x} \psi_{0}(s, t) d s\right)\right]\right\|_{L^{\infty}} \\
& \leq C\left\|\partial_{x}^{k} \psi_{1}(x, t)\right\|_{L^{\infty}}+C \sum_{j=0}^{k-1}\left\|\partial_{x}^{j} \psi_{1}(x, t)\right\|_{L^{\infty}}\left\|\partial_{x}^{k-j-1} \psi_{0}(x, t)\right\|_{L^{\infty}} \\
& \quad \leq C t^{-\frac{1}{2}-\frac{k+1}{2}}+C \sum_{j=0}^{k-1} t^{-\frac{1}{2}-\frac{k-j-1}{2}} t^{-\frac{j+2}{2}}=C t^{-\frac{1}{2}-\frac{k+1}{2}} .
\end{aligned}
$$

It is easy to check that

$$
\left\|\partial_{x}^{k}\left(\psi_{0}(x, t) \int_{-\infty}^{x} \psi_{1}(s, t) d s\right)\right\|_{L^{\infty}} \leq C t^{-\frac{1}{2}-\frac{k+1}{2}}
$$


Next, using induction as before, for the first ratio $J_{21}(x, t)$ in (3.34) we obtain the estimate

$$
\left\|\partial_{x}^{k} J_{21}(t)\right\|_{L^{\infty}} \leq C t^{-\frac{1}{2}-\frac{k+1}{2}} .
$$

The numerator of the second ratio $J_{22}(x, t)$ in (3.35) satisfies the inequality

$$
\left\|\partial_{x}^{k} \int_{-\infty}^{x} \delta_{0}(s, t) d s\right\|_{L^{2}} \leq C t^{-\frac{1}{4}-\frac{k}{2}}
$$

Using induction, we prove that

$$
\left\|\partial_{x}^{k}\left[\frac{\int_{-\infty}^{x} \delta_{0}(s, t) d s}{\left(1-\int_{-\infty}^{x} \psi_{0}(s, t) d s\right)}\right]\right\|_{L^{2}} \leq C t^{-\frac{1}{4}-\frac{k}{2}}
$$

As a result,

$$
\left\|\partial_{x}^{k} J_{2}(x, t)\right\|_{L^{2}} \leq C \sum_{j=0}^{k} t^{-\frac{1}{2}-\frac{j+1}{2}} t^{-\frac{1}{4}-\frac{k-j}{2}} \leq C t^{-\frac{1}{4}-\frac{k+2}{2}} .
$$

Thus, estimates (3.33) and (3.36) imply

$$
\left\|\partial_{x}^{k}\left(u(t)-u_{0}(t)-u_{1}(t)\right)\right\|_{L^{2}} \leq C t^{-\frac{1}{4}-\frac{k+2}{2}} \log (1+t) .
$$

Now, as in Lemma 2, it remains to write the function $\left(\partial_{x}^{k}\left(u(t)-u_{0}(t)-u_{1}(t)\right)\right)^{2}$ in the form of an integral with variable upper limit and apply the Cauchy-Bunyakovskiu inequality:

$$
\begin{aligned}
& \left\|\partial_{x}^{k}\left(u(t)-u_{0}(t)-u_{1}(t)\right)\right\|_{L^{\infty}} \\
& \quad \leq C\left\|\partial_{x}^{k}\left(u(t)-u_{0}(t)-u_{1}(t)\right)\right\|_{L^{2}}^{1 / 2}\left\|\partial_{x}^{k+1}\left(u(t)-u_{0}(t)-u_{1}(t)\right)\right\|_{L^{2}}^{1 / 2} \\
& \quad \leq C t^{-\frac{k+3}{2}} \log (1+t), \quad t>1 .
\end{aligned}
$$

The theorem is proved.

\section{References}

[1] S. Kawashima and Y. Tanaka, Stability of rarefaction waves for a model system of a radiating gas, Kyushu J. Math. 58 (2004), 211-250. MR2117245(2005k:35264)

[2] K. Hamer, Nonlinear effects on the propagation of sound waves in a radiating gas, Quart. J. Mech. Appl. Math. 24 (1971), 155-168.

[3] K. Ito, BV-Solution of a hyperbolic-elliptic system for a radiating gas, Hokkaido Univ., Preprint, no. 368, 1997, 33 pp.

[4] Y. Liu and S. Kawashima, Asymptotic behavior of solutions to a model system of a radiating gas, Commun. Pure Appl. Anal. 10 (2011), no. 1, 209-223. MR2746535(2012d:35248)

[5] C. Lattanzio and P. Marcati, Global well-posedness and relaxation limits of a model for radiating gas, J. Differential Equations 190 (2003), no. 2, 439-465. MR 1970037 (2004c:35259)

[6] P. Laurencot, Asymptotic self-similarity for a simplified model for radiating gases, Asymptot. Anal. 42 (2005), no. 3-4, 251-262. MR2138795 (2006d:35230)

[7] A. A. Solov'ev, The leading term of the asymptotics of the solution of the Hamer equation, Dokl. Akad. Nauk 439 (2011), no. 6, 740-742; English transl., Dokl. Math. 84 (2011), no. 1, 555-557. $\operatorname{MR} 2883803$

[8] D. Serre, $L^{1}$-stability of constants in a model for radiating gases, Commun. Math. Sci. 1 (2003), no. 1, 197-205. MR1979850 (2004c:76127)

Mathematics Department, Chelyabinsk State University, ul. Brat'ev Kashirinykh 129, CheLYABINSK 454001, RUSSIA

E-mail address: alsol@csu.ru

Received 18/MAR/2013

Translated by A. PLOTKIN 